\title{
NARMA-L2 Control of a Nonlinear Half-Car Servo-Hydraulic Vehicle Suspension System
}

\section{Jimoh Pedro, John Ekoru}

School of Mechanical, Aeronautical and Industrial Engineering, University of the Witwatersrand, Private Bag 03, WITS2050, Johannesburg, South Africa e-mail: Jimoh.Pedro@wits.ac.za; John.Ekoru@students.wits.ac.za

\begin{abstract}
In this paper, the performance of a nonlinear, 4 degrees-of-freedom, servohydraulic half-car active vehicle suspension system is compared with that of a passive vehicle suspension system with similar model parameters. The active vehicle suspension system is controlled by an indirect adaptive Neural Network-based Feedback Linearization controller (NARMA-L2). Hydraulic actuator force tracking is guaranteed by an inner Proportional+Integral+Derivative-based force feedback control loop. The output responses of the vehicles are presented and analyzed in the frequency and time domains, in the presence of model uncertainties in the form of variation in vehicle sprung mass loading. The results show that the NARMA-L2-based active vehicle suspension system performed better than the passive vehicle suspension system within the constraints.
\end{abstract}

Keywords: Neural Networks; NARMA-L2; PID Control; Nonlinear Vehicle Suspension System; Hydraulic Actuator Dynamics; Model Uncertainty

\section{Introduction}

Vehicle Suspension Systems (VSS) are highly nonlinear uncertain systems [1]. In VSS design, a tolerable compromise between conflicting vehicle parameters (ride comfort, handling and road holding) must be found, within the limits of suspension travel $[1 ; 2]$. VSS design is an active area of research involving the development of Passive Vehicle Suspension Systems (PVSS), Semi-Active Vehicle Suspension Systems (SAVSS) and Active Vehicle Suspension Systems (AVSS) $[3 ; 4]$. The rapid progress within this field can be attributed to advances made in optimal control and in computer processing power and to the increasing affordability of actuators and sensors [2; 3]. Compared with PVSS and SAVSS, AVSS are better at addressing the VSS design trade-off; PVSS and SAVSS can only dissipate forces incident on the VSS, whereas AVSS introduce forces into the VSS. However, AVSS are more complex and consume greater amounts of energy [5]. 
Examples of control techniques succesfully applied to AVSS include: PID control [6], optimal control [2; 7], robust control methods such as $H_{2}$ [8], $H_{\infty}$ [9], $H_{2} / H_{\infty}$ [10], linear parameter varying (LPV) [11], nonlinear control techniques like sliding mode control (SMC) [12], backstepping control [13] and feedback linearization (FBL) [14]. Intelligent control techniques such as fuzzy logic control (FLC) [15] and various Neural Network (NN)-based control methods [1; 16; 17] have also been applied.

Neglecting actuator dynamics in the study of AVSS has restricted the amount of experimental validation possible in the past $[18 ; 19]$. In research works where actuator dynamics are considered, hydraulic actuators are most often selected because they have a fast response time, high stiffness, a superior power-to-weight ratio, low cost and low heat dissipation during periods of sustained force generation, compared to other actuators [1; 17]. However, hydraulic actuators are highly nonlinear and prone to chattering in AVSS applications [18]. Backpressure caused by tight coupling between motion of the vehicle body and actuator force generation requires the use of hydraulic actuator force feedback [19]. Actuator force feedback improves vehicle ride comfort and road holding by stabilizing the hydraulic actuator, and guaranteed desired forces levels are attained. In the literature, force control is commonly applied utilizing a system of control loops; i.e., actuator force feedback control in the inner loop and sprung mass displacement control in the outer loop $[14 ; 18 ; 19]$.

In industry, PID control is the most common control method employed (it is simple in structure, and tuning is straightforward) [20]. PID control has been applied both to benchmark AVSS performance [17;21] and as the main control method [6]. However, its lack of robustness to parameter variation and the requirement of high loop gains have motivated research with the aim of enhancing PID controller performance for AVSS applications [22; 23; 24].

Highly nonlinear systems can be transformed into linear systems to enable the application of linear control methods by making use of FBL [25]. The implementation of FBL may prove difficult as feedback of all system states is required, and issues may arise regarding the robustness of the linearized system. This is solved by a combination of FBL with intelligent control methods [1]. In Buckner et al. [16], radial basis function (RBF) NNs were trained to estimate the nonlinear suspension damping and spring forces in order to cancel out nonlinear suspension dynamics by using NNFBL and to control a quarter-car AVSS with a linear electro-mechanical force actuator. Offline training of the NNs was performed using input-output data obtained from a quarter-car suspension test-rig. The RBF NN weights were updated online. Pedro and Dahunsi [1] proposed a Multi Layer Perceptron (MLP) NNFBL controller for a nonlinear, electrohydraulic quarter-car AVSS. MLP NNs were trained to approximate the nonlinear functions using the Levenberg-Marquardt (LM) algorithm. Compared with a PIDcontrolled AVSS, the NNFBL-controlled AVSS performed better at tracking a square-wave suspension travel reference signal, consuming a lower amount of energy. 
The exact values of the VSS model parameters such as sprung mass loading, suspension damping and suspension spring stiffness, etc. are uncertain. Therefore, consideration should be given to include acceptably bounded parametric uncertainty in the design of control systems for VSS $[4 ; 9 ; 26 ; 27 ; 28]$.

In this paper, the effects of the uncertainties in vehicle sprung mass loading on the performance of AVSS and PVSS are explored. A NNFBL-controlled nonlinear half-car AVSS, with PID hydraulic actuator force feedback control and a nonlinear half-car PVSS with similar model parameters are compared and their performance is analyzed in the frequency and time domain. This paper is organized as follows: the mathematical model of the nonlinear, half-car AVSS with hydraulic actuator dynamics is presented in Section 2, followed by the performance specifications in Section 3. The overall control architecture and design are given in Section 4. In Section 5, simulation results are presented and discussed and the paper is concluded in Section 6.

\section{System Model}

\subsection{Modelling Assumptions}

The following simplifying assumptions are made during the mathematical modelling [8]:

1) All joints connecting the suspension system components are considered to be ideal.

2) The vehicle is moving in a straight line in the horizontal direction at a constant velocity. Forces and moments due to cornering, accelerating and braking of the vehicle are neglected.

3) Both the sprung and unsprung masses are assumed to be uniform in mass.

4) Sprung mass loading does not vary with time [4]. However, different sprung mass loadings are applied to test controller's robustness under parameter variations.

5) The vehicle body is taken as rigid.

6) Road surface roughness and irregularities are the sole source of vehicular vibration; road surface elastic deformation and engine-induced vibrations are ignored $[2 ; 4]$.

7) Non-uniformity of the tyre as well as wheel unbalance effects are disregarded.

8) The sprung mass centre of mass rests along the vehicle body's longitudinal centreline. 
9) The sensors and actuators used respond instantly to changes in measured parameters.

10) Heaving and pitching are taken as the main vehicle body movements. Other possible motions such as yawing, rocking and lateral motions of the sprung mass centre of gravity about the nominal travel path are neglected.

11) The simulation is carried out at the point of dynamic equilibrium. Therefore, the weight of the vehicle is ignored.

12) The vehicle body pitch angular displacement, $\theta$, is varied through large angles about the point of equilibrium [29].

13) Tyre damping couples the wheel and the vehicle body motion at the wheelhop frequency and thus is not ignored [10].

\subsection{Physical and Mathematical Modelling}

Figure 1 illustrates a half-car AVSS physical model of sprung mass $M s$, pitch moment of inertia $I_{\theta}$ and pitch angular displacement $\theta$, front and rear unsprung masses $m u_{f}$ and $m u_{r}$, respectively.

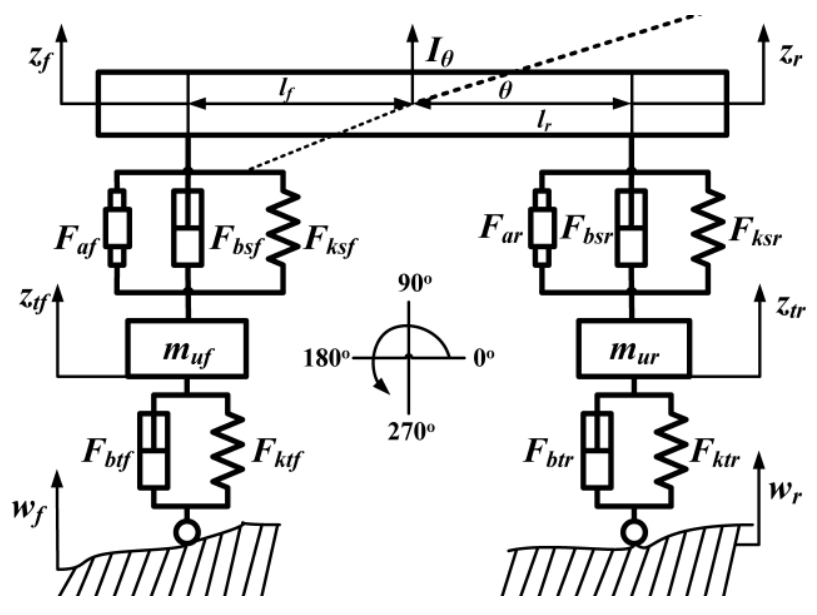

Figure 1

Schematic of a half-car AVSS

$z_{C}, z_{t f}$ and $z_{t r}$ are the vertical displacements of the sprung mass at the centre of gravity, the front tyre and the rear tyre, respectively. The lengths between the front and rear axles and the vehicle centre of gravity are given by $l_{f}$ and $l_{r}$, respectively. The front and rear suspension travels are expressed as $y_{f}=z_{t f}-\left(z_{C}-l_{f} \sin \theta\right)$ and $y_{r}=z_{t r}-\left(z_{C}+l_{r} \sin \theta\right)$, respectively. 
The application of Newton's $2^{\text {nd }}$ law of motion to the nonlinear half-car AVSS gives the governing equations of motion in state-space form as $[7 ; 11 ; 29 ; 30]$ :

$\dot{\mathbf{x}}=\mathbf{f}(\mathbf{x})+\mathbf{g}(\mathbf{x}) \mathbf{u}+\mathbf{p w}$

where $\mathbf{x}$ is the state vector, $u$ is the control input vector, $\mathbf{w}$ is the disturbance input vector, $\mathbf{f}(\mathbf{x})$ is the system vector, $\mathbf{g}(\mathbf{x})$ is the control input matrix and $\mathbf{p}$ is the disturbance input matrix.

$$
\begin{aligned}
\mathbf{x} & =\left[\mathrm{z}_{\mathrm{c}}, \theta, \mathrm{z}_{\mathrm{tf}}, \mathrm{z}_{\mathrm{tr}}, \dot{\mathrm{z}}_{\mathrm{c}}, \dot{\theta}, \dot{\mathrm{z}}_{\mathrm{tf}}, \dot{\mathrm{z}}_{\mathrm{tr}}, \mathrm{P}_{\mathrm{lf}}, \mathrm{P}_{\mathrm{lr}}, \mathrm{x}_{\mathrm{vf}}, \mathrm{x}_{\mathrm{vr}}\right]^{\mathrm{T}} \\
& =\left[x_{1}, x_{2}, x_{3}, x_{4}, x_{5}, x_{6}, x_{7}, x_{8}, x_{9}, x_{10}, x_{11}, x_{12}\right]^{\mathrm{T}} \\
\mathbf{u} & =\left[\mathrm{u}_{1}, \mathrm{u}_{2}\right]^{\mathrm{T}}=\left[v_{f}, v_{r}\right]^{\mathrm{T}} \\
\mathbf{w} & =\left[w_{f}, w_{r}, \dot{w}_{f}, \dot{w}_{r}\right]^{\mathrm{T}} \\
\mathrm{f} & =\left[f_{1}, f_{2}, f_{3}, f_{4}, f_{5}, f_{6}, f_{7}, f_{8}, f_{9}, f_{10}, f_{11}, f_{12}\right]^{\mathrm{T}}
\end{aligned}
$$

The output equation is given as:

$$
\mathbf{y}=\left[\begin{array}{c}
y_{f} \\
y_{r}
\end{array}\right]=\mathbf{h}(\mathbf{x})=\left[\begin{array}{l}
x_{3}-x_{1}+l_{f} \sin x_{2} \\
x_{4}-x_{1}+l_{r} \sin x_{2}
\end{array}\right]
$$

The components of vector $\mathbf{f}(\mathbf{x}), \mathbf{g}(\mathbf{x})$ and $\mathbf{p}$ are given in [29].

\subsection{Road Input Disturbance Modelling}

Equations 18 and 19 express the front and rear wheel input disturbances, $w_{f}$ and $w_{r}$, respectively.

$w_{f}= \begin{cases}0.5 a(1-\cos (2 \pi V t / \lambda)) & 1 \leq t \leq 1+\lambda / V \\ 0 & \text { otherwise }\end{cases}$

$w_{r}= \begin{cases}0.5 a(1-\cos (2 \pi V t / \lambda)) & 1+t_{d} \leq t \leq 1+t_{d}+\lambda / V \\ 0 & \text { otherwise }\end{cases}$

where $a$ is the bump amplitude, $V$ is the vehicle forward velocity, $\lambda$ is the disturbance wavelength, and $t$ is the simulation time. $t_{d}$ is the time delay between the front and rear wheels written as:

$t_{d}=\left(l_{f}+l_{r}\right) / V$

The bump profile is illustrated in Fig. 2. The half-car, hydraulic actuator and road input disturbance model parameters are given in $[11 ; 25 ; 29]$. 


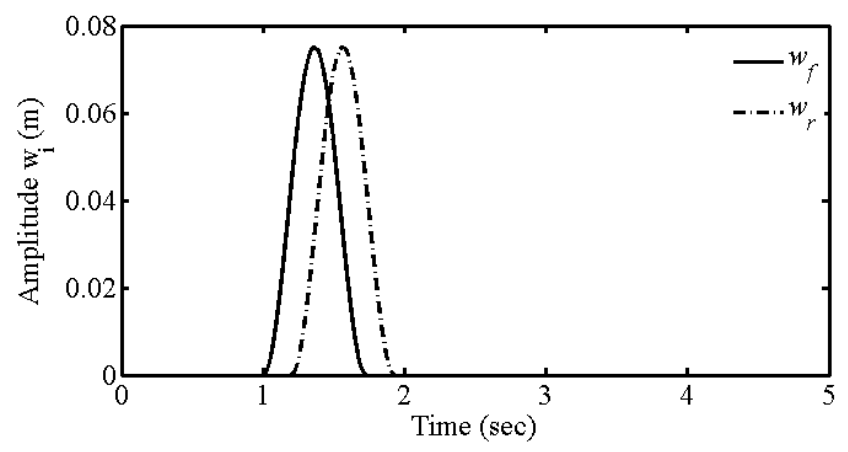

Figure 2

Bump road input disturbance

\section{Performance Specifications}

The performance specifications utilized in this work are:

1) The closed loops should be nominally stable and the controller must possess good command following and disturbance rejection.

2) The maximum allowable suspension travel should not exceed the limit given as:

$\left|y_{i}\right| \leq z_{\max }$

where $i \in(f, r) . z_{\max }$ is the maximum suspension travel, equal to $0.08 m$.

3) The maximum allowable control voltage, $u_{\max }$, is expressed as:

$\left|u_{i}(t)\right| \leq u_{\max }$

where $u_{\max }$ is the maximum allowable control voltage equal to $10 \mathrm{~V}$.

4) The maximum allowable controlled force, $F_{a i}$, is given as:

$\left|F_{a i}\right| \leq \pm M s \times g$

where $g$ is the acceleration due to gravity, equal to $9.81 \mathrm{~m} / \mathrm{s}^{2}$.

5) To maintain good road holding, the dynamic tyre load, $F_{t i}$, should not exceed the static load, $F_{t i}^{\text {stat }}$ [4]:

$F_{t i} \leq F_{t i}^{\text {stat }}$

where 


$$
\begin{aligned}
& F_{t i}=k t_{i}\left(\dot{z}_{t i}-\dot{w}_{i}\right)+b t_{i}\left(\dot{z}_{t i}-\dot{w}_{i}\right) \\
& F_{t i}^{\text {stat }}=g\left[M s \times l_{i} /\left(l_{f}+l_{r}\right)+m u_{i}\right]
\end{aligned}
$$

6) The RMS values of the performance parameters will be used to enable detailed performance comparison of the AVSS with the PVSS. For $n$ simulation samples:

$$
\Theta_{R M S}=\sqrt{n^{-1} \sum_{0}^{n}(\Theta)^{2}}
$$

where

$$
\Theta=\left[y_{i}, F_{t i}, \ddot{z}_{c}, \ddot{\theta}, u_{i}, F_{a i}\right]^{T} \text {. }
$$

7) Ride Comfort: The evaluation of vehicle ride comfort is based on the ISO 2631-1 frequency weighted RMS acceleration [31]. $W_{k}$, the ISO 2631-1 frequency weighting for acceleration input at the feet, is selected since theVSS models do not include vehicle seats. A fifth order approximation of $W_{k}$ is expressed as [32]:

$$
\begin{aligned}
W_{k}(s)= & \left(87.72 s^{4}+1138 s^{3}+11336 s^{2}+5453 s+5509\right) \div\left(s^{5}+92.6854 s^{4}\right. \\
& \left.+2549.83 s^{3}+25969 s^{2}+81057 s+79783\right)
\end{aligned}
$$

The weighted RMS acceleration, $a_{w i}^{R M S}$, for $n$ samples is given by:

$$
a_{w i}^{R M S}=\sqrt{n^{-1} \sum_{0}^{n}\left(k_{a x i s} W_{k}\left(\ddot{z}_{c}\right)\right)^{2}}
$$

where the axis multiplication factor $k_{\text {axis }}=0.40$ for vertical sprung mass acceleration along the $z$ axis. A vibration induced discomfort scale for various values of $a_{w i}^{R M S}$ is given in [31].

\section{Controller Design}

\subsection{Control Architecture}

The AVSS control configuration shown in Fig. 3 consists of two control loops: an outer NNFBL suspension travel feedback control loop and an inner PID hydraulic actuator force feedback control loop. Suspension travel is chosen as the controlled output variable on the outer loop because it is easily measured with displacement transducers [15]. 


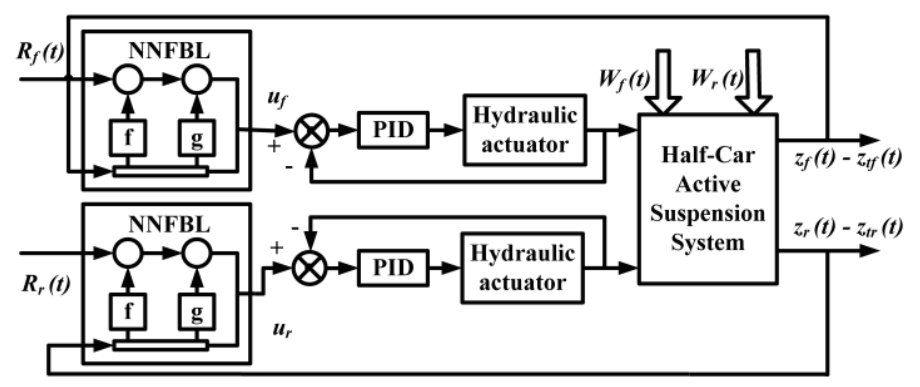

Figure 3

Control architecture

\subsection{PID Force Control Loop Design}

The PID force control input, $u_{i}$ to the half-car AVSS actuators is given in Equation 31:

$u_{i}=K_{P i} e_{i}(t)+K_{I i} \int e_{i}(t) d t+K_{D i} d\left(e_{i}(t)\right) / d t$

$e_{i}(t)=F_{\text {airef }}(t)-F_{a i}(t)$

where $K_{P}$ is the proportional gain, $K_{I}$ is the integral gain, $K_{D}$ is the derivative gain, and $e_{i}$ is the error between the desired actuator force reference signal, $F_{\text {airef }}$, and the actual actuator force, $F_{a i}$. The PID controller gains obtained by the Ziegler-Nichols tuning method are $K_{P f}=K_{\mathrm{Pr}}=0.0010, K_{I f}=K_{I r}=0.0145$ and $K_{D f}=K_{D r}=0.0003$.

\subsection{NNFBL Suspension Travel Control Loop Design}

FBL involves the cancellation of nonlinear system dynamics, thereby enabling the application of linear control techniques to highly nonlinear systems [1; 24]. A system expressed in state-space form can be linearized with an input $\mathbf{u}$ [33]:

$$
\begin{aligned}
& \dot{\mathbf{x}}=\mathbf{f}(\mathbf{x})+\mathbf{g}(\mathbf{x}) \mathbf{u} \\
& \mathbf{u}=\mathbf{g}(\mathbf{x})^{-1}\left[-\mathbf{f}(\mathbf{x})-\mathbf{K}^{\mathbf{T}} \mathbf{x}+\mathbf{R}\right]
\end{aligned}
$$

where $\mathbf{K}$ is a matrix of controller gains and $\mathbf{R}$ is the reference input. Substituting equation (34) into (33) cancels out the nonlinear dynamics of the system, $\mathbf{f}(\mathbf{x})$ and $\mathbf{g}(\mathbf{x})$. This gives a linear system able to track reference trajectory using a linear controller expressed as [33]:

$\dot{\mathbf{x}}=-\mathbf{K}^{\mathbf{T}} \mathbf{x}+\mathbf{R}$ 
Using NNs to approximate $\mathbf{f}(\mathbf{x})$ and $\mathbf{g}(\mathbf{x})$, equation (34) becomes [43]: $\mathbf{u}=\mathbf{g}_{\mathbf{N N}}(\mathbf{x})^{-1}\left[-\mathbf{f}_{\mathrm{NN}}(\mathbf{x})-\mathbf{k}^{\mathbf{T}} \mathbf{x}+\mathbf{R}\right]$

where $\mathbf{f}_{\mathrm{NN}}(\mathbf{x})$ and $\mathbf{g}_{\mathrm{NN}}(\mathbf{x})$ are the $\mathrm{NN}$ approximations of $\mathbf{f}(\mathbf{x})$ and $\mathbf{g}(\mathbf{x})$, respectively. The system is required to track a reference model [33]:

$\dot{\mathbf{x}}_{\mathbf{m}}=-\mathbf{K}^{\mathbf{T}} \mathbf{x}_{\mathbf{m}}+\mathbf{R}$

Substituting equation (36) into equation (33) gives [33]:

$\dot{\mathbf{x}}=\mathbf{f}(\mathbf{x})+\left(\mathbf{g}(\mathbf{x}) / \mathbf{g}_{\mathrm{NN}}(\mathbf{x})\right)\left[-\mathbf{f}_{\mathrm{NN}}(\mathbf{x})-\mathbf{k}^{\mathbf{T}} \mathbf{x}+\mathbf{R}\right\rfloor$

The controller error $\mathbf{e}$ is calculated as [33]:

$\mathbf{e}=x-x_{m}$

The error dynamics given by [33]:

$$
\dot{\mathbf{e}}=-\mathbf{K}^{\mathbf{T}} \mathbf{e}+\left[\mathbf{f}(\mathbf{x})-\mathbf{f}_{\mathrm{NN}}(\mathbf{x})\right]+\left[\mathbf{g}(\mathbf{x})-\mathbf{g}_{\mathrm{NN}}(\mathbf{x})\right] \mathbf{u}
$$

The MATLAB/Simulink ${ }^{\circledR}$ NARMA-L2 control toolbox was used to perform NNFBL on the outer control loop. Typically, there are two steps involved in indirect adaptive control using NNs: system identification and control design. Since this tool is a SISO tool, two separate NARMA-L2 controllers were used (the half-car AVSS is a MIMO system). Since the NARMA-L2 controller is a SISO tool, the AVSS model was modified to allow for collection of the Input/Output (I/O) data required for system identification; by grounding and terminating part of the AVSS inputs and outputs, the AVSS appears as SISO to the controller (see Fig. 4). The system identification was then carried out in four steps: (a) Experimentation, (b) Model Structure Selection, (c) Model Estimation, and (d) Model Validation.

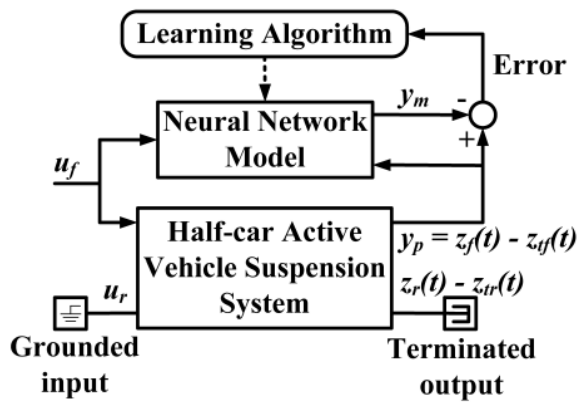

Figure 4

System identification of the half-car AVSS for NNFBL control 


\subsubsection{Experimentation}

Two random non-saturating input signals covering the entire operational range of the half-car AVSS were used to generate the I/O data. The data, $Z_{i}^{n}$, was collected in the form presented in Equation 41 in which $u_{i}(k)$ and $y_{i}(k)$ are the input and output to the system, respectively, $k$ is the number of the sampling instant, and $n$ is the total number of samples taken [34; 35] (see Figure 4).

$$
Z_{i}^{n}=f\left\{\left[u_{i}(k), y_{i}(k)\right] ; k=1, \ldots, n\right\}
$$

\subsubsection{Model Structure Selection}

The NARMA models given in Equation 42, in which $d$ is the system delay, $n a$ is the number of past inputs, $n b$ is the number of past outputs and $F$ is a nonlinear function, were trained to present the half-car AVSS model's forward dynamics. The nonlinear function $F$ is approximated by the NN during identification [34; 35].

$$
\begin{aligned}
y_{i}(k+d)= & F\left[y_{i}(k), y_{i}(k-1), \ldots y_{i}(k-n a+1), u_{i}(k), u_{i}(k-1), \ldots,\right. \\
& \left.u_{i}(k-n b+1)\right]
\end{aligned}
$$

FBL involves cancellation of the nonlinear system dynamics. The NARMA-L2 controller achieves this by training two MLP NN to approximate the nonlinear functions $\mathbf{f}(\mathbf{x})$ and $\mathbf{g}(\mathbf{x})$. The MLP NN models have two layers. The first layer (hidden layer) contains neurons with tangent-sigmoid activation function [34; 35]:

$$
\tan \operatorname{sig}(n)=(2) /\left(1+e^{-2(n)}\right)-1=\left(1-e^{-2(n)}\right) /\left(1+e^{-2(n)}\right)
$$

The second layer (output layer) contains linearly activated neurons. Both the hidden and output layers contain a bias. The companion form is expressed in Equation 44. This form enables tracking of reference signal $y_{r i}(k+d)$. However, because $y_{i}(k)$ is required to calculate $u_{i}(k)$ and both occur at the same sampling instant, the resulting controller (see Eq. 37) is not practically feasible [34; 35].

$$
\begin{gathered}
\hat{y}_{i}(k+d)=f\left[y_{i}(k), y_{i}(k-1), \ldots y_{i}(k-n+1), u_{i}(k), u_{i}(k-1), \ldots,\right. \\
\left.u_{i}(k-n a+1)\right]+g\left[y_{i}(k), y_{i}(k-1), \ldots, y_{i}(k-n+1),\right. \\
\left.u(k-1), \ldots, u_{i}(k-n b+1)\right] \cdot u_{i}(k) \\
u_{i}(k)=\left[\hat{y}_{r i}(k+d)-f\left[y_{i}(k), y_{i}(k-1), \ldots y_{i}(k-n+1), u_{i}(k),\right.\right. \\
\left.u_{i}(k-1), \ldots, u_{i}(k-n a+1)\right] \times\left[g \left[y_{i}(k), y_{i}(k-1), \ldots,\right.\right. \\
\left.\left.y_{i}(k-n+1), u(k-1), \ldots, u_{i}(k-n b+1)\right]\right]^{-1}
\end{gathered}
$$

By setting the plant delay $d \geq 2$ with a model order $n=n a=n b=2$, a NARMA model and therefore a practical NARMA-L2 controller can be developed as given in Equations 46 and 47, respectively. 
Fig. 5 illustrates the NARMA-L2 controller implementation $[34 ; 35]$.

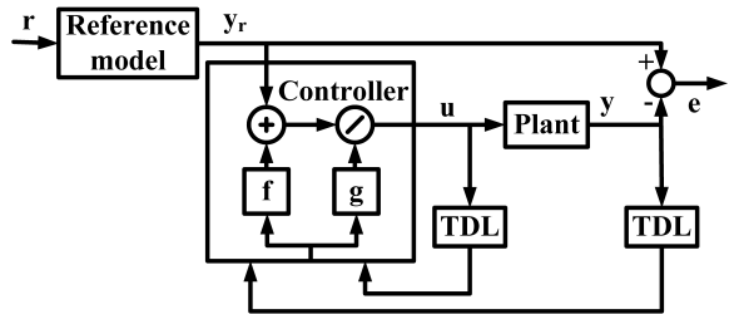

Figure 5

NARMA-L2 Controller implementation

$$
\begin{gathered}
y_{i}(k+d)=f\left[y_{i}(k), y_{i}(k-1), \ldots y_{i}(k-n+1), u_{i}(k), u_{i}(k-1), \ldots,\right. \\
\left.u_{i}(k-n+1)\right]+g\left[y_{i}(k), y_{i}(k-1), \ldots, y_{i}(k-n+1),\right. \\
\left.u(k-1), \ldots, u_{i}(k-n+1)\right] \cdot u_{i}(k+1) \\
u_{i}(k)=\left[y_{r i}(k+d)-f\left[y_{i}(k), \ldots y_{i}(k-n+1), u_{i}(k), u_{i}(k-1), \ldots,\right.\right. \\
\left.u_{i}(k-n+1)\right] \times\left[g \left[y_{i}(k), y_{i}(k-1), \ldots, y_{i}(k-n+1),\right.\right. \\
\left.\left.u(k-1), \ldots, u_{i}(k-n+1)\right]\right]^{-1}
\end{gathered}
$$

The plant model order is determined by evaluating the Lipschitz quotients of the I/O data, plotting the model order index against the lag space, i.e., the number of past inputs and outputs [17; 23]. Figs. (a) and (b) show that the slopes of both graphs increase at the "knee point" where the number of past inputs and outputs is greater than or equal to two. Therefore, setting a model order greater than two could cause overfitting of data when training the NNs [17; 23].

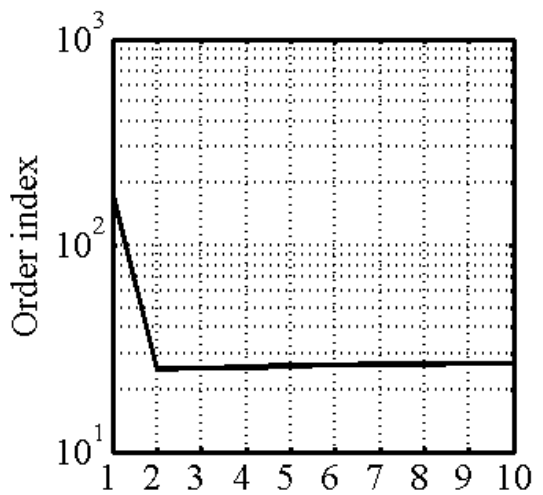

Number of past inputs and outputs

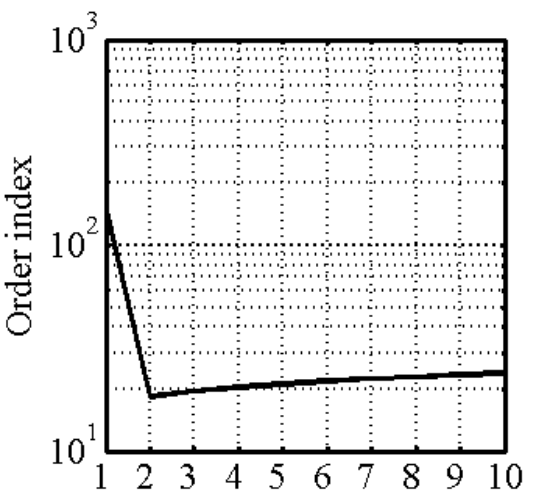

Number of past inputs and outputs

(a) Front suspension

(b) Rear suspension

Figure 6

Two dimensional plot of the Order of Index versus lag space 


\subsubsection{Model Estimation}

Model estimation involves using the $\mathrm{I} / \mathrm{O}$ data collected to train an $\mathrm{NN}$ to approximate the desired function. The NNs were trained using backpropagation method, by minimizing the Mean Square Error (MSE) [35]. For an AVSS plant output $\mathbf{y}_{\mathbf{i}}$ and NN model output $\mathbf{y}_{\mathbf{m i}}$, the NN model MSE is calculated as follows:

$$
M S E=n^{-1} \sum_{0}^{n}\left(e_{i}\right)^{2}=n^{-1} \sum_{0}^{n}\left(\mathbf{y}_{\mathbf{i}}-\mathbf{y}_{\mathbf{m i}}\right)^{2}
$$

where $e_{i}$ is the error between the AVSS plant output and the NN model output and $n$ is the number of training samples. The Levenberg-Marquardt (LM) algorithm was used for training the NNs, producing MSEs of $1.6736 \times 10^{-7}$ after 13 training epochs and $3.8147 \times 10^{-7}$ after 28 training epochs for the front and rear suspension NNs, respectively. The NNFBL parameters for both the Front and Rear Suspension are listed in Table 2 for a maximum number of 1000 training epochs. The LM algorithm produced the best training results compared to the other training algorithms given in Table 3 for the rear suspension.

Table 2

NNFBL parameters for front and rear suspension

\begin{tabular}{|l|c|}
\hline Parameters & Value \\
\hline Number of hidden layer neurons & 5 \\
\hline Number of delayed plant inputs & 2 \\
\hline Number of delayed plant outputs & 2 \\
\hline Sampling interval $T_{s}$ & $0.001 \mathrm{sec}$ \\
\hline Normalize training data & No \\
\hline Plant input range & {$[-5000 N: 5000 N]$} \\
\hline Plant output range & {$[-0.08 m: 0.08 m]$} \\
\hline Maximum interval value & $0.5 \mathrm{sec}$ \\
\hline Minimum interval value & $0.1 \mathrm{sec}$ \\
\hline Number of training samples & 10000 \\
\hline
\end{tabular}

\subsubsection{Levenberg-Marquardt (LM)}

The LM algorithm is an algorithm that enables rapid training of NNs by calculating an approximation of the Hessian matrix, $H$, rather than the actual Hessian matrix [35]. For performance functions expressed in the form of a sum of squares, such as the MSE, the Hessian matrix can be approximated as [35]:

$H=J^{T} J$ 
where $J$ is the Jacobian matrix containing the first derivatives of the NN errors with respect to the NN weights and biases. The gradient, $G$, is given by [35]:

$G=J^{T} e$

where $e$ is a vector of $\mathrm{NN}$ errors. At sampling instant $k$, the vector of the NN weights and biases, $x_{k}$, is updated using the approximation of the Hessian matrix [35]:

$x_{k+1}=x_{k}-\left(J^{T} e\right) /\left(J^{T} J+\mu I\right)=x_{k}-G /(H+\mu I)$

where $I$ is the identity matrix and $\mu$ is a scalar parameter that is adjusted at each sampling instant to ensure that the performance function is minimized [45].

Table 3

Performance of NNFBL training algorithms for rear suspension

\begin{tabular}{|l|c|c|}
\hline \multirow{2}{*}{\multicolumn{1}{|c|}{ Full name of algorithm }} & \multicolumn{2}{c|}{ Rear suspension } \\
\cline { 2 - 3 } & $\begin{array}{c}\text { No. of } \\
\text { epochs }\end{array}$ & MSE \\
\hline $\begin{array}{l}\text { Broyden-Fletcher-Goldfarb-Shanno (BFGS) quasi- } \\
\text { Newton Backpropagation }\end{array}$ & 24 & $5.0564 \times 10^{-3}$ \\
\hline Bayesian Regulation & 32 & $1.1742 \times 10^{-3}$ \\
\hline Powell-Beale conjugate gradient backpropagation & 9 & $7.5448 \times 10^{-4}$ \\
\hline Fletcher-Powell conjugate gradient backpropagation & 10 & 0.25491 \\
\hline Polak-Ribiereconjugate gradient backpropagation & 4 & 0.054292 \\
\hline Gradient descent backpropagation & 1000 & $7.24 \times 10^{-3}$ \\
\hline Gradient descent with momentum backpropagation & 6 & 2.16 \\
\hline Gradient descent with adaptive learning backpropagation & 33 & 3.3688 \\
\hline $\begin{array}{l}\text { Gradient with momentum and adaptive learning } \\
\text { backpropagation }\end{array}$ & 48 & 0.24551 \\
\hline Levenberg-Marquardt backpropagation & 28 & $3.8147 \times 10^{-7}$ \\
\hline One step secant backpropagation & 27 & $9.6757 \times 10^{-4}$ \\
\hline Resilient backpropagation & 39 & $8.6774 \times 10^{-4}$ \\
\hline Scaled conjugate gradient backpropagation & 29 & 0.039014 \\
\hline
\end{tabular}

\subsubsection{Model Validation}

$50 \%$ of the I/O data is used for $\mathrm{NN}$ training, $25 \%$ for validation and the remaining $25 \%$ for testing of the $\mathrm{NN}$ models. 


\section{Simulation Results and Discussion}

The VSS models were built in the MATLAB/ Simulink ${ }^{\circledR}$ environment. The fixed step solver ODE-3 (Bogacki-Shampine) was utilized, with the sampling time $T_{s}=0.0001 \mathrm{sec} . T_{s}$ is smaller than the fastest half-car AVSS model dynamics, enabling observation of all model dynamics [1]. Robustness to parameter variation was tested by varying $M s$ and $I_{\theta}$ by $\pm 20 \%$ about their nominal values $[9 ; 36]$.

\subsection{Frequency Domain Results}

A frequency sweep method similar to that used by [37] was applied here, by use of a chirp road input disturbance signal of amplitude $\pm 15 \mathrm{~mm}$ [38]. Its frequency was set to vary between $0-100 \mathrm{~Hz}$ over a $100 \mathrm{sec}$ period, in order to expose the vehicle to a wide range of input disturbance frequencies. Spectral analysis of the AVSS and PVSS model outputs was performed utilizing the MATLAB Welch algorithm/spectral estimator [39]. The "Hamming" window setting with a segment length of $n / 100$ ( $n$ is the total number of samples) and a percentage overlap of $2^{14}$ produced optimal frequency response plots. Due to space contrainsts, only the results for the rear suspension travel will be shown.

Figs. 7(a) and 7(b) show the AVSS and PVSS rear suspension travels Power Spectral Density (PSD) responses. The magnitude of the AVSS response at the "knee" between $1 \mathrm{~Hz}$ and the body-hop frequency $(\approx 2 \mathrm{~Hz})$ is smaller than that of the PVSS. In the frequency range below $4 \mathrm{~Hz}$ and between $4-8 \mathrm{~Hz}$, both the AVSS and PVSS perform similarly. At the wheel-hop frequency $(\approx 12 \mathrm{~Hz})$, the magnitude of the AVSS response is smaller than that of the PVSS. At high frequencies $(>20 \mathrm{~Hz})$, the AVSS and PVSS behave similarly.

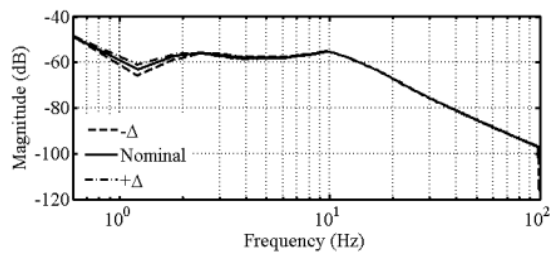

(a) AVSS

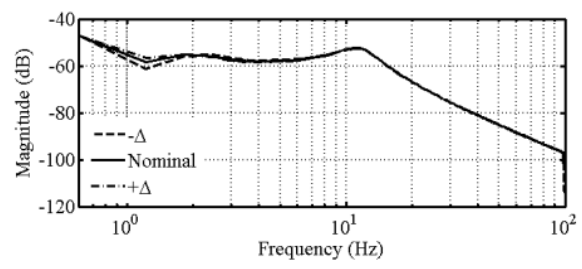

(b) PVSS

Figure 7

Rear suspension travel PSD

Figs. 8(a) and 8(b) show the AVSS and PVSS sprung mass acceleration PSD response, respectively. At low frequencies (below $4 \mathrm{~Hz}$ ), the AVSS is less sensitive to variation in $M s$ and $I_{\theta}$ than the PVSS. The response of the AVSS about wheel-hop frequency $(\approx 12 \mathrm{~Hz})$ appears more rounded than that of the PVSS. Above $20 \mathrm{~Hz}$, both the AVSS and PVSS behave similarly. 


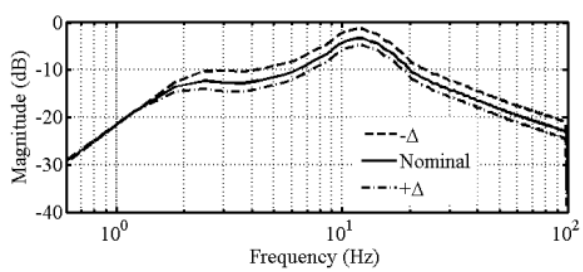

(a) AVSS

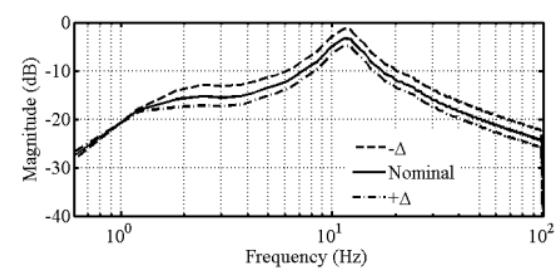

(b) PVSS

Figure 8

Sprung mass acceleration PSD

Figs. 9(a) and 9(b) give the AVSS and PVSS pitch angular acceleration PSD responses, respectively. At frequencies less than $4 \mathrm{~Hz}$, the AVSS is less sensitive than the PVSS to variations in $M s$ and $I_{\theta}$, although the magnitude of the AVSS response is greater than that of the PVSS within this frequency range. In the $4-8 \mathrm{~Hz}$ frequency range, around the wheel-hop frequency and above $20 \mathrm{~Hz}$, the AVSS and PVSS behave similarly.

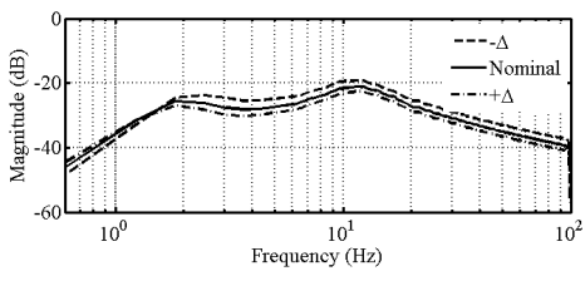

(a) AVSS

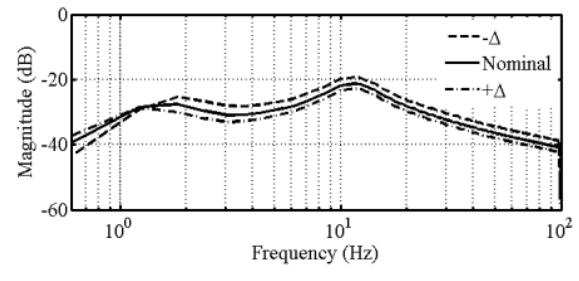

(b) PVSS

Figure 9

Pitch angular acceleration PSD

\subsection{Time Domain Results}

Due to space contrainsts, only the results for the rear suspension will be shown. Table 4 contains the RMS values obtained as the VSS models traversed the road input disturbance given in Section 2.2 for suspension travel regulation $\left(R_{i}(t)=0\right)$, at a constant forward velocity. Although the AVSS performance is significantly better than that of the PVSS, the RMS results for both the front and rear suspension (see Table 4) indicate that an increase in vehicle sprung mass causes a reduction in AVSS suspension travel performance. The minimum and maximum peak front and rear suspension travel values obtained by the AVSS are also lower than those of the PVSS. An increase in vehicle mass causes the suspension travel workspace to reduce. Fig. 10 suggests that the AVSS is less likely to hit the suspension travel limits $( \pm 0.08 m)$ than the PVSS as the mass of the vehicle increases. 
Table 4

RMS values for $\Delta= \pm 20 \%\left\{M s, I_{\theta}\right\}$ about their nominal values

\begin{tabular}{|c|c|c|c|c|c|c|c|c|c|}
\hline \multirow[b]{2}{*}{ Parameters } & \multicolumn{3}{|c|}{$-\Delta$} & \multicolumn{3}{|c|}{ Nominal } & \multicolumn{3}{|c|}{$+\Delta$} \\
\hline & $\underset{\tilde{D}}{\tilde{2}}$ & $\begin{array}{l}\infty \\
\frac{2}{4}\end{array}$ & 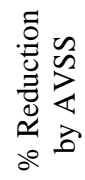 & $\begin{array}{l}\tilde{2} \\
\boldsymbol{D}^{2}\end{array}$ & $\begin{array}{l}\mathscr{2} \\
\stackrel{2}{2}\end{array}$ & 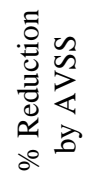 & $\begin{array}{l}\tilde{n} \\
\tilde{2} \\
2\end{array}$ & \begin{tabular}{l}
$\approx$ \\
\multirow{2}{*}{} \\
$\geq$
\end{tabular} & 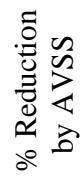 \\
\hline $\begin{array}{l}\text { Rear } \\
\text { Suspension } \\
\text { Travel }(m)\end{array}$ & 0.0069 & 0.0047 & 31.88 & 0.0085 & 0.0059 & 30.59 & 0.0099 & 0.0069 & 30.3 \\
\hline $\begin{array}{c}\text { Rear } \\
\text { Dynamic } \\
\text { Tyre } \\
\text { Force }(N)\end{array}$ & 222.03 & 228.15 & -3.69 & 262.29 & 268.35 & -2.31 & 302.09 & 305.28 & -1.06 \\
\hline $\begin{array}{c}\text { Sprung Mass } \\
\text { Acceleration } \\
\left(m / s^{2}\right)\end{array}$ & 1.0753 & 0.8285 & 23.2 & 1.1293 & 0.8658 & 23.33 & 1.1395 & 0.8673 & 23.89 \\
\hline $\begin{array}{c}\text { Pitch } \\
\text { Angular } \\
\text { Acceleration } \\
\left(\mathrm{rad} / \mathrm{s}^{2}\right)\end{array}$ & 0.6453 & 0.6166 & 4.45 & 0.5849 & 0.5559 & 4.96 & 0.5231 & 0.4944 & 5.49 \\
\hline $\begin{array}{c}\text { Rear } \\
\text { Actuator } \\
\text { Voltage }(V)\end{array}$ & - & 0.1585 & - & - & 0.1845 & - & - & 0.2073 & - \\
\hline $\begin{array}{c}\text { Rear } \\
\text { Actuator } \\
\text { Force }(N)\end{array}$ & - & 77.934 & - & - & 95.713 & - & - & 112.3 & - \\
\hline
\end{tabular}

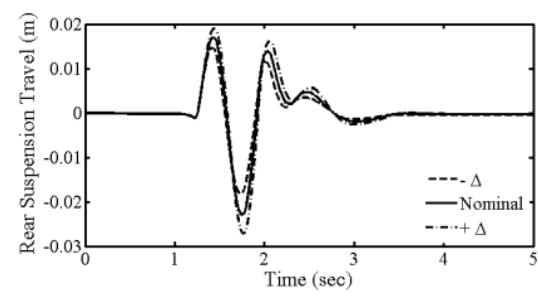

(a) AVSS

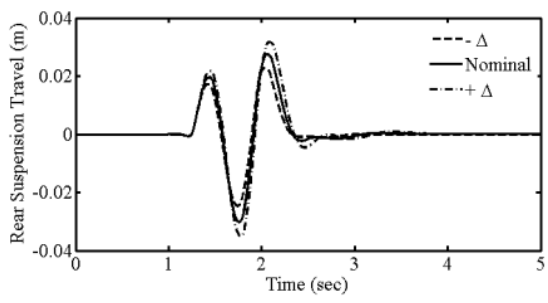

(b) PVSS

Figure 10

Rear suspension travel 
The AVSS and PVSS sprung mass acceleration are shown in Fig. 11. Although both the AVSS and PVSS maintained a "Not Uncomfortable" level of discomfort throughout, Table 4 shows that the AVSS reduced the ISO 2631-1 weighted RMS acceleration better than the PVSS. With regards to pitch angular acceleration, the RMS values in Table 4 indicate that AVSS performance deteriorated with an increase in vehicle mass. The AVSS peak pitch angular acceleration values tend to be higher when compared with the corresponding PVSS values. However, Fig. 12 shows the AVSS dampens oscillation approximately $0.5 \mathrm{sec}$ faster than the PVSS.

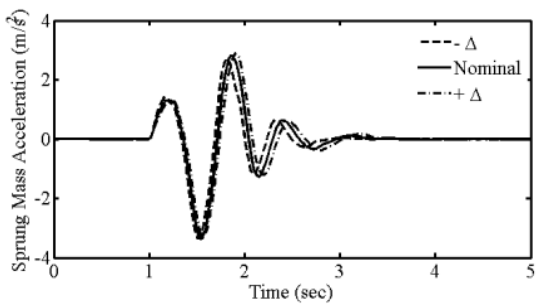

(a) AVSS

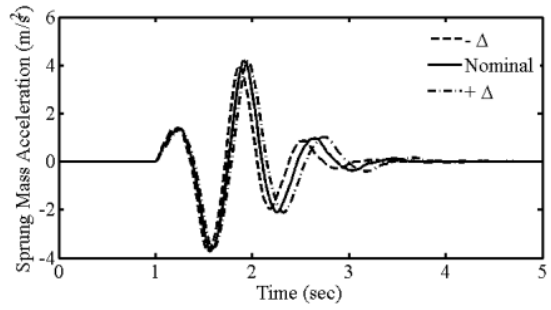

(b) PVSS

Figure 11

Sprung mass acceleration

Table 4

Weighted RMS acceleration and discomfort levels

\begin{tabular}{|c|c|c|c|c|c|}
\hline \multirow{2}{*}{} & \multicolumn{2}{|c|}{ PVSS } & \multicolumn{2}{c|}{ AVSS } & \\
\cline { 2 - 6 } & $\begin{array}{c}\text { Weighted RMS } \\
\text { Acceleration } \\
a_{w i}^{R M S}\left(\mathrm{~m} / \mathrm{s}^{2}\right)\end{array}$ & $\begin{array}{c}\text { ISO 2631-1 } \\
\text { Level of } \\
\text { Discomfort }\end{array}$ & $\begin{array}{c}\text { Weighted RMS } \\
\text { Acceleration } \\
a_{w i}^{R M S}\left(\mathrm{~m} / \mathrm{s}^{2}\right)\end{array}$ & $\begin{array}{c}\text { ISO 2631-1 } \\
\text { Level of } \\
\text { Discomfort }\end{array}$ & $\begin{array}{c}\% \\
\text { Reduction } \\
\text { by AVSS }\end{array}$ \\
\hline$-\Delta$ & 0.2117 & N.U. & 0.1663 & N.U. & 21.45 \\
\hline Nominal & 0.2205 & N.U. & 0.1714 & N.U. & 22.27 \\
\hline$-\Delta$ & 0.2217 & N.U. & 0.1703 & N.U. & 23.18 \\
\hline
\end{tabular}

where N.U. = Not Uncomfortable

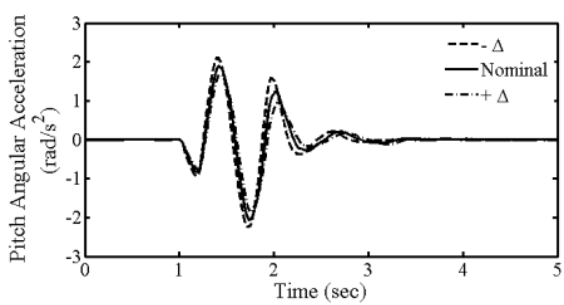

(a) AVSS

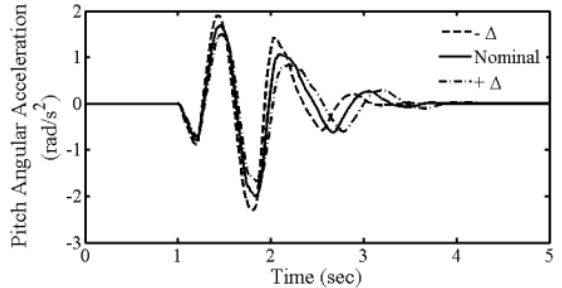

(b) PVSS

Figure 12

Pitch angular acceleration 


\subsection{Effect of the Inner Force Control Loop}

To investigate the effect of the inner force control loop on AVSS performance, an AVSS with both inner PID force control loop and outer NARMA-L2 suspension travel control loop is compared with a second AVSS without the inner force control loop. The sprung mass acceleration time domain responses of both AVSS are plotted against each other for nominal $M s$ and $I_{\theta}$. As shown in Fig. 13, the AVSS with force control is able to dampen the sprung mass oscillation due to the road input disturbance faster than the one without force control, by stabilizing the hydraulic actuator.

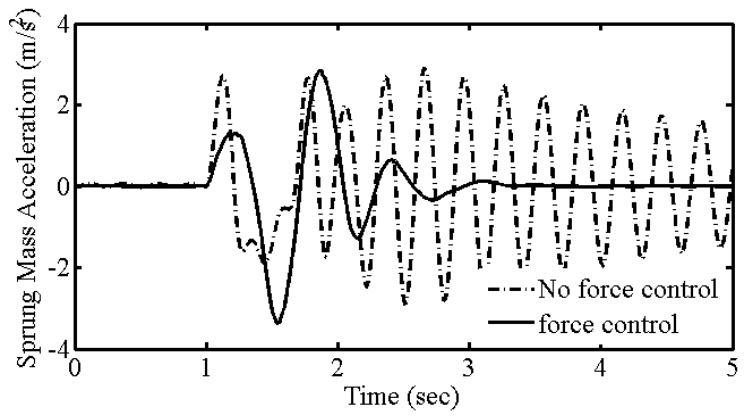

Figure 13

AVSS sprung mass acceleration with and without force control

\section{Conclusion}

In this work, a NNFBL controlled nonlinear AVSS with PID based hydraulic actuator force feedback has been presented to improve suspension system model of the AVSS. Robustness to parameter variation was tested by varying the performance. Two-layer tansig activated MLP NNs were used to identify the NN vehicle sprung mass loading and the moment of inertia by $\pm 20 \%$, comparing the AVSS and PVSS performance in the frequency and time domains. The frequency domain results showed that the AVSS possesses greater robustness to uncertainties than the PVSS, particularly at frequencies below $4 \mathrm{~Hz}$ and around the wheel-hop frequency. In the time domain, the overall AVSS performance in terms of reducing RMS parameters was better than that of the PVSS. The AVSS suspension travel was significantly lower than the PVSS suspension travel, both remaining within the specified limits throughout. The AVSS and PVSS did not exceed the specified front and rear dynamic tyre force limits. The AVSS maintained the actuator force well below the specified limits.

\section{References}

[1] Pedro, J. and Dahunsi, O. (2011). Neural Network-based Feedback Linearization Control of a Servo-Hydraulic Vehicle Suspension System, International Journal of Applied Mathematics and Computer Science 21(1): $137-147$ 
[2] Hrovat, D. (1997). Survey of Advanced Suspension Developments and Related Optimal Control Applications, Automatica 33(10): 1781-1817

[3] Guglielmino, E. and Edge, K. A. (2004). A Controlled Friction Damper for Vehicle Applications, Control Engineering Practice 12(4): 431-443

[4] Gao, H., Lam, J. and Wang, C. (2006). Multi-Objective Control of Vehicle Active Suspension Systems via Load-Dependent Controllers, Journal of Sound a nd Vibration 290(3-5): 654-675

[5] Fischer, D. and Isermann, R. (2004). Mechatronic Semi-Active and Active Vehicle Suspensions, Control Engineering Practice 12(11): 1353-1367

[6] Guclu, R. (2003). Active Control of Seat Vibrations of a Vehicle Model Using Various Suspension Alternatives, Turkish Journal of Engineering and Environmental Sciences 27(6): 361-373

[7] Hassanzadeh, I., Alizadeh, G., Shirjoposht, N. P. and Hashemzadeh, F. (2010). A New Optimal Nonlinear Approach to Half Car ACTIVE Suspension, IACSIT International Journal of Engineering and Technology 2(1): $78-84$

[8] Pedro, J. O. (2007). $H_{2}$ - LQG/LTR Controller Design for Active Suspension Systems, R and D Journal of the South African Institution of Mechanical Engineering 23(2): 32-41

[9] Chen, H., Liu, Z. Y. and Sun, P. Y. (2005). Application of Constrained $H_{\infty}$ Control to Active Suspension Systems on Half-Car Models, Journal of Dynamic Systems, Measurement and Control, Transactions of the ASME 127(3): 345-354

[10] Akcay, H. and Turkay, S. (2009). Influence of Tire Damping on Mixed $\mathrm{H}_{2} / \mathrm{H}_{\infty}$ Synthesis of Half-Car Active Suspensions, Journal of Sound and Vibration 322(1-2): 15-28

[11] Szaszi, I., Gáspár, P. and Bokor, J. (2002). Nonlinear Active Suspension Modelling Using Linear Parameter Varying Approach, Proceedings of the $10^{\text {th }}$ Mediterranean Conference on Control and Automation (MED2002), Lisbon, Portugal, pp. 1-10

[12] Yoshimura, T., Kume, A., Kurimoto, M. and Hino, J. (2001). Construction of an Active Suspension System of a Quarter-Car Model Using the Concept of Sliding Mode Control, Journal of Sound and Vibration 239(2): 187-199

[13] Yagiz, N. and Hacioglu, Y. (2008). Backstepping Control of a Vehicle with Active Suspensions, Control Engineering Practice 16(12): 1457-1467

[14] Fateh, M. M. and Alavi, S. S. (2009). Impedance Control of an Active Suspension System, Mechatronics 19(1): 134-140 
[15] Du, H. and Zhang, N. (2009a). Static Output Feedback Control for Electrohydraulic Active Suspensions via T-S Fuzzy Model Approach, Journal of Dynamic Systems, Measurement and Control: Transactions of ASME 131(5): 1-11

[16] Buckner, G. D., Schuetze, K. T. and Beno, J. H. (2000). Active Vehicle Suspension Control Using Intelligent Feedback Linearization, Proceedings of the American Control Conference, Vol. 6, Chicago, IL, USA, pp. 40144018

[17] Dahunsi, O. A. and Pedro, J. O. (2010). Neural Network-based Identification and Approximate Predictive Control of a Servo-Hydraulic Vehicle Suspension System, Engineering Letters 18(4): 357-368

[18] Chantranuwathana, S. and Peng, H. (2004). Adaptive Robust Force Control for Vehicle Active Suspension, International Journal of Adaptive Control and Signal Processing 18(2): 83-102

[19] Sam, Y. M. and Hudha, K. (2006). Modelling and Force Tracking of Hydraulic Actuator for an Active Suspension System, Proceedings of the IEEE conference on Industrial Electronics and Applications, (ICIEA 2006), Singapore, Singapore, pp. 1-6

[20] Astrom, K. J. and Hagglund, T. (2001). The future of PID control, Control Engineering Practice 9(11): 1163-1175

[21] Eski, I. and Yildirim, S. (2009). Vibration Control of Vehicle Active Suspension System Using a New Robust Neural Network Control System, Simulation Modelling Practice and Theory 17(5): 778-793

[22] Feng, J. Z., Li, J. and Yu, F. (2003). GA-based PID and Fuzzy Logic Control for Active Vehicle Suspension System, International Journal of Automotive Technology 4(4): 181-191

[23] Dahunsi, O. A., Pedro, J. O. and Nyandoro, O. T. (2010). System Identification and Neural Network-based PID Control of Servo-Hydraulic Vehicle Suspension System, SAIEE Africa Research Journal, Research Journal of the South African Institute of Electrical Engineers 101(3): 93105

[24] Precup, R.-E., Preit, S., Petriu, E. M., Tar, J. K., Tomescu, M. L. and Pozna, C. (2009). Generic Two-Degree-of-Freedom Linear and Fuzzy Controllers for Integral Processes, Journal of the Franklin Institute 346(10) 980-1003

[25] Deng, J., Becerra, V. and Stobart, R. (2009). Input Constraints Handling in an MPC/Feedback Linearization Scheme, International Journal of Applied Mathematics and Computer Science 19(2): 219-232 
[26] Du, H. and Zhang, N. (2009b). Fuzzy Control for Nonlinear Uncertain Electrohydraulic Active Suspensions with Input Constraint, IEEE Transactions on Fuzzy Systems 17(2): 343-356

[27] Gáspár, P., Szabó, Z. and Bokor, J. (2012). LPV Design of Fault-Tolerant Control for Road Vehicles, International Journal of Applied Mathematics and Computer Science 22(1): 173-182

[28] Rodić, A. and Mester, Gy. (2011). The Modeling and Simulation of an Autonomous Quad-Rotor Microcopter in a Virtual Outdoor Scenario, Acta Polytechnica Hungarica 8(4): 107-122

[29] Huang, C., Lin, J. and Chen, C. (2010). Road Adaptive Algorithm Design of Half-Car Active Suspension System, Expert Systems with Applications 37(6): 4392-4402

[30] Ekoru, J. E. D., and Pedro, J. O. (2012). Intelligent Feedback Linearizationbased Control of Half-Car Active Suspension Systems, Proceedings of the fifth IASTED Africa International Conference on Modelling and Simulation (AfricaMS 2012), Gaborone, Botswana, pp. 161-168

[31] International Organization for Standardization (1997). Mechanical Vibration and Shock - Evaluation of Human Exposure to Whole Body Vibration (Part 1: General Requirements), (ISO 2631-1:1997) second edition, International Standards, Geneva, Switzerland

[32] Zuo, L. and Nayfeh, S. (2003). Low Order Continuous-Time filters for Approximation of the ISO 2631-1 Human Vibration Sensitivity Weightings, Journal of Sound and Vibration 265(2): 459-465

[33] Hagan, M. T., Demuth, H., B. (1999). Neural Networks for Control, Proceedings of the 1999 American Control Conference, Vol. 3 pp. 16421656, San Diego, CA

[34] Pedro, J. O., Dahunsi, O. A. and Nyandoro, O. T. C. (2012). Direct Adaptive Neural Control of Antilock Braking Systems Incorporated with Passive Suspension Dynamics, Journal of Mechanical Science and Technology 26(12): 4115-4130

[35] Beale, M. H., Hagan, M. T. and Demuth, H. B. (2010). Neural Network Toolbox ${ }^{\text {TM }} 7$ User's Guide, The MathWorks, Inc., Natick, MA

[36] Du, H., Zhang, N. and Lam, J. (2008). Parameter-Dependent Input-delayed Control of Uncertain Vehicle Suspensions, Journal of Sound and Vibration 317(3-5): 537-556

[37] Savaresi, S. M., Poussot-Vassal, C., Spelta, C., Sename, O. and Dugard, L. (2010). Semi-Active Suspension Control Design for Vehicles, ButterworthHeinemann, Boston 
[38] Sammier, D., Sename, O. and Dugard, L. (2003). Skyhook and $H_{\infty}$ Control of Semi-Active Suspensions: Some Practical Aspects, Vehicle System Dynamics 39(4): 279-308

[39] The MathWorks Inc. (2012). Signal Processing Toolbox for use with Matlab ${ }^{\mathbb{B}}$ User's Guide version 6.17, Natick, MA 